\section{RSP}

http://www.rsp.fsp.usp.br/
Revista de Saúde Pública

\title{
Fatores associados ao near miss neonatal no Brasil
}

\author{
Theonas Gomes Pereira' (iD, Daniele Marano da Rocha" (iD, Vânia Matos Fonseca" (iD, Maria \\ Elisabeth Lopes Moreira" ID, Silvana Granado Nogueira da Gama"l' iD \\ I Centro Universitário UNINOVAFAPI. Curso de Nutrição. Teresina, PI, Brasil \\ " Fundação Oswaldo Cruz. Instituto Nacional de Saúde da Mulher, da Criança e do Adolescente Fernandes \\ Figueira. Pós-Graduação em Saúde da Criança e da Mulher. Rio de Janeiro, RJ, Brasil \\ III Fundação Oswaldo Cruz. Escola Nacional de Saúde Pública. Departamento de Epidemiologia em Métodos \\ Quantitativos em Saúde. Rio de janeiro, RJ, Brasil
}

\section{RESUMO}

OBJETIVO: Este estudo avalia a associação entre fatores sociodemográficos, características maternas, organização dos serviços de saúde e near miss neonatal em maternidades públicas e privadas do Brasil.

MÉTODOS: Trata-se de uma coorte prospectiva de nascidos vivos da pesquisa Nascer no Brasil, realizada entre 2011 e 2012. As variáveis foram estabelecidas a partir da literatura e organizadas em três níveis: distal, intermediário e proximal. A avaliação foi realizada a partir dos resultados das análises bivariadas e de seus respectivos valores-p, com nível de significância $<0,20$, pelo teste de Wald. Para a análise multivariada, foram inseridas as variáveis contidas no nível distal, conservadas no modelo quando significativas $(\mathrm{p}<0,05)$. O mesmo foi feito no ajuste dos níveis intermediário e proximal.

RESULTADOS: No nível distal, nenhuma variável apresentou associação significativa com o desfecho. No nível intermediário, idade da mãe maior ou igual a 35 anos (risco relativo $\mathrm{RR}=1,32$; IC95\% 1,04-1,66), parto cesáreo ( $\mathrm{RR}=1,34$; IC95\% 1,07-1,67), uso de fumo (RR = 1,48; IC95\% 1,04-2,10), síndrome hipertensiva gestacional (RR = 2,29; IC95\% 1,98-3,14), diabetes pré-gestacional ( $\mathrm{RR}=2,63$; IC95\% 1,36-5,05) e gestação gemelar $(\mathrm{RR}=2,98$; IC95\% 1,90-4,68) foram variáveis associadas ao desfecho. No nível proximal, o pré-natal não adequado ( $\mathrm{RR}=1,71$; IC95\% 1,36-2,16) e a localização do hospital/maternidade na capital (RR = 1,89; IC95\% 1,40-2,55) foram associados ao near miss neonatal.

CONCLUSÃO: Os resultados mostram que o near miss neonatal foi influenciado pelas variáveis da organização dos serviços de saúde e pelas características maternas.

DESCRITORES: Near miss. Complicações na Gravidez. Fatores de Risco. Fatores Socioeconômicos. Serviços de Saúde Materno-Infantil.

originais sejam creditados.

\footnotetext{
Como citar: Pereira TG, Marano

DR, Fonseca VM, Gama SGN

Moreira MEL. Fatores associados ao

near miss neonatal no Brasil. Rev

Saude Publica. 2020;54:123.

Copyright: Este é um artigo de acesso aberto distribuído sob os termos da Licença de Atribuição Creative Commons, que permite uso irrestrito, distribuição e reprodução em qualquer meio, desde que o autor e a fonte

Theonas Gomes Pereira

Rua José Olímpio de Melo, 3426

E-mail: theonasgp@yahoo.com.br

Recebido: 12 fev 2020

Aprovado: 29 mai 2020
} 


\section{INTRODUÇÃO}

O conceito de near miss neonatal é recente, sendo definido como eventos mórbidos que quase resultam em morte de recém-nascidos $(\mathrm{RN})$ nos primeiros 28 dias de vida ${ }^{1,2}$. Posto que na literatura existem distintas definições para o near miss neonatal ${ }^{2-4}$, este estudo considerou o conceito adotado por Silva et al. ${ }^{2}$, que, no ano de 2014, avaliaram os dados da pesquisa Nascer no Brasil - um estudo nacional de base hospitalar e nível regional - com o objetivo de definir as variáveis que poderiam predizer a mortalidade neonatal e compor o indicador near miss neonatal. Após serem testadas 19 variáveis, 5 foram escolhidas, a saber: peso ao nascer $<1.500 \mathrm{~g}$, Apgar no quinto minuto de vida $<7$, uso de ventilação mecânica $(\mathrm{VM})$, idade gestacional < 32 semanas e relato de más-formações congênitas. Os autores do estudo supracitado ${ }^{2}$ avaliaram que esse indicador tem elevada sensibilidade (92,5\%), especificidade (97,1\%) e acurácia (97\%), o que confere robustez à sua utilização e à monitorização desse agravo.

Os critérios definidos por Silva et al. ${ }^{2}$ foram validados pelos estudos conduzidos por Kale et al. ${ }^{3}$ e França et al..$^{4}$ No primeiro estudo ${ }^{3}$, uma coorte de nascidos vivos em duas capitais brasileiras, foram utilizados três critérios pragmáticos de Silva et al. ${ }^{2}$ para a definição do near miss neonatal: peso ao nascer $<1.500$ g, idade gestacional $<32$ semanas e escore de Apgar $<7$ no quinto minuto de vida. No segundo estudo ${ }^{4}$, também uma coorte de nascidos vivos, foram utilizados dados dos Sistemas de Informação em Saúde, selecionando-se as variáveis utilizadas no estudo de Kale et al. ${ }^{3}$, acrescidas de internação em unidade de terapia intensiva (UTI) neonatal e de más-formações congênitas. Ambos os estudos evidenciaram a acurácia da proposta de Silva et $_{\text {al. }}{ }^{2}$

O indicador near miss neonatal possui inúmeras vantagens por ser uma ferramenta para identificar fatores de risco associados ao óbito neonatal e monitorar mudanças na morbimortalidade neonatal ${ }^{5}$. Dentre essas vantagens, cita-se a identificação de morbidades graves e de suas causas primárias, o que pode reduzir o óbito neonatal e permite que o indicador seja usado em diversas configurações para identificar problemas no sistema de saúde - configurando-se como instrumento de gestão - e, se for o caso, para instituir ações corretivas $^{2}$, redundando em melhoria da qualidade da assistência neonatal ${ }^{5}$.

Em relação aos óbitos infantis no Brasil, observa-se que esse desfecho ocorre preponderantemente no período neonatal (70\%), sobretudo nas primeiras semanas de vida (54\%) ${ }^{6}$. Logo, há uma redução da mortalidade infantil no período pós-neonatal (de 23,1 para 9,5 por mil nascidos vivos) ${ }^{6}$.

Diante disso, vários autores têm discutido a mortalidade neonatal ${ }^{6,7}$; no entanto, são escassos os estudos que se detiveram na análise dos principais fatores associados ao near miss neonatal ${ }^{5,8}$. O avanço no conhecimento da rede de fatores de risco maternos envolvidos na mortalidade neonatal (idade, escolaridade ${ }^{6}$, situação conjugal, hábito de fumar e uso do álcool ${ }^{9}$, doenças prévias e atuais da gestação, adequação do pré-natal, entre outros) ${ }^{10}$, com base na estratégia de modelagem hierárquica para discriminar as relações entre determinantes do near miss neonatal, pode ser útil na sua avaliação; outrossim, possibilita apontar ações necessárias ao aperfeiçoamento da assistência, com consequente impacto nos desfechos neonatais.

Portanto, este estudo tem como objetivo avaliar a associação entre os fatores sociodemográficos, as características maternas, a organização dos serviços de saúde e o near miss neonatal em hospitais públicos e privados representativos das cinco regiões do Brasil.

\section{MÉTODOS}

Esta pesquisa consiste em uma coorte prospectiva de nascidos vivos constituída de informações dos questionários aplicados às puérperas e de dados coletados dos prontuários 
de pacientes que participaram da pesquisa Nascer no Brasil. A coleta de dados ocorreu entre fevereiro de 2011 e outubro de 2012. Detalhes em relação à amostra são encontrados no estudo de Vasconcellos et al..$^{11} \mathrm{e}$, sobre o método, em Leal et al. ${ }^{12}$

Para a construção da variável dependente deste estudo, o near miss neonatal, foi utilizada a classificação do estudo de Silva et al. ${ }^{2}$, que selecionou cinco variáveis associadas à mortalidade neonatal: peso ao nascer $<1.500 \mathrm{~g}$, índice de Apgar $<7$ no quinto minuto de vida, uso de VM, idade gestacional < 32 semanas e presença de más-formações congênitas. Sendo assim, todos os RN que sobreviveram ao período neonatal e apresentaram pelo menos um dos preditores citados foram considerados casos de near miss neonatal ${ }^{2}$.

Foram amostrados $24.200 \mathrm{RN}$, sendo 23.837 deles nascidos vivos, 128 natimortos, 171 óbitos neonatais e 64 óbitos neonatais resgatados do Sistema de Informações sobre Mortalidade (SIM). Os casos de óbitos neonatais após a alta hospitalar foram obtidos por meio de um questionário aplicado após o $42^{\circ}$ dia de internação da mulher ou no $28^{\circ}$ dia de internação do RN. Informações mais detalhadas sobre o método podem ser obtidas no estudo de Silva et al${ }^{2}$.

O modelo hierárquico do near miss neonatal foi baseado em fatores de risco para o óbito do $\mathrm{RN}^{13}$. Ressalta-se que as condições de saúde do $\mathrm{RN}$ e da atenção neonatal são inerentes à definição do near miss neonatal (idade gestacional, peso ao nascer, escore de Apgar, entre outros). Por isso, foram consideradas no nível proximal as variáveis relativas à organização do serviço de saúde. As variáveis independentes foram organizadas por nível de proximidade com o desfecho, inserindo-se primeiro as do nível distal e, em seguida, as dos níveis intermediário e proximal, estabelecidas a partir da literatura ${ }^{1,2,6,8}$ e organizadas em um modelo teórico conceitual (Figura).

No nível distal, foram incluídos os aspectos sociodemográficos: região (Sudeste, Norte, Nordeste, Centro-Oeste ou Sul); escolaridade materna em anos completos (ensino fundamental incompleto, ensino fundamental completo, ensino médio completo ou ensino superior completo); classe econômica (A+B, C ou D+E); cor da pele (branca ou preta/parda/amarela/indígena); $\mathrm{e}$ chefe de família (não ou sim). Foi considerada "chefe de família" quando a mãe era a pessoa de referência para tomada de decisões na família ${ }^{11}$, e a classificação econômica foi baseada nos critérios da Associação Brasileira de Empresas de Pesquisa (Abep) ${ }^{14}$.

No nível intermediário foram incluídas as variáveis representativas das características maternas: idade materna (12 a 19 anos, 20 a 34 anos, ou maior ou igual a 35 anos); situação conjugal (sem companheiro ou com companheiro); primiparidade (não ou sim); tipo de parto (vaginal, com fórceps ou cesáreo); tabagismo materno, considerando o uso habitual de fumo após o quinto mês da gravidez (não ou sim); síndrome hipertensiva da gestação (não ou sim); sífilis (não ou sim); diabetes pré-gestacional (não ou sim); diabetes gestacional (não ou sim);

Nível distal

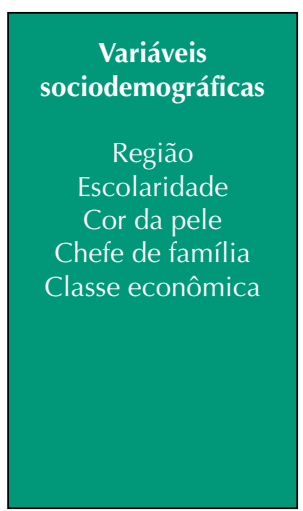

Nível intermediário

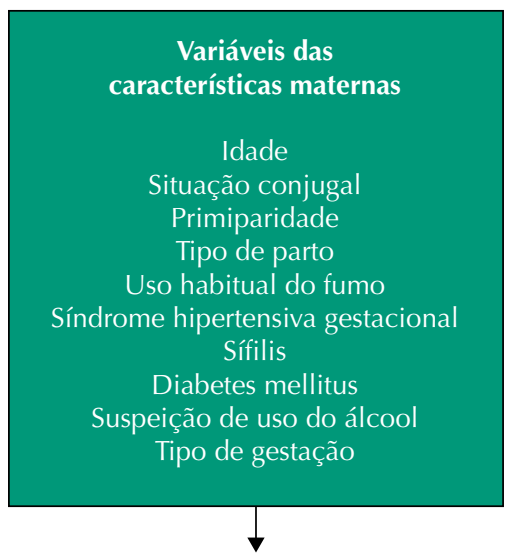

Nível proximal

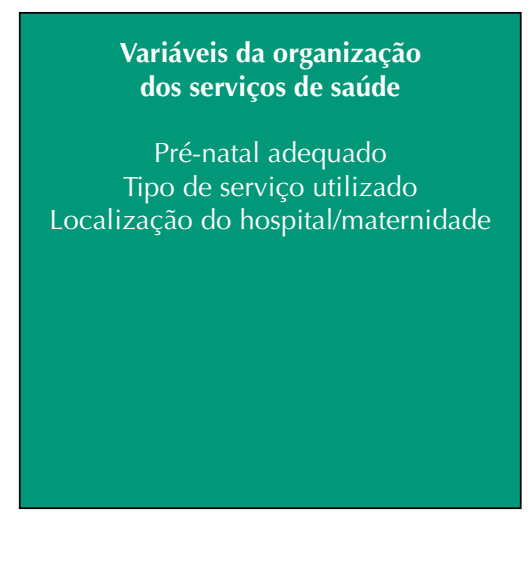

Figura. Modelo teórico-conceitual dos fatores preditores do near miss neonatal no Brasil. 
suspeição de uso inadequado de álcool (não ou sim); e tipo de gestação (única ou gemelar). Para mensurar o tabagismo, a variável fumar após o quinto mês de gestação foi considerada de maior risco para o baixo peso no $\mathrm{RN}^{15}$. Quanto à suspeição de uso de álcool, utilizou-se o questionário T-ACE (acrônimo das palavras inglesas: tolerance, annoyed, cut down e eye-opener), composto por quatro questões principais, às quais é atribuída uma pontuação, sendo o valor máximo do questionário igual a cinco (a primeira questão vale até dois pontos e, da segunda à quarta pergunta, a valoração é de até um ponto). A pontuação total maior ou igual a dois indica um caso positivo, ou seja, a mãe éidentificada como consumidora de álcool ${ }^{16}$. A variável síndrome hipertensiva da gestação se refere ao diagnóstico de hipertensão crônica, hipertensão gestacional, pré-eclâmpsia, eclâmpsia ou síndrome de Hellp .

No nível proximal foram consideradas as variáveis relacionadas à organização do serviço de saúde: pré-natal adequado (não ou sim); tipo de serviço utilizado no pré-natal (público ou privado); e localização do hospital/maternidade (não capital ou capital). Considerou-se assistência pré-natal adequada aquela iniciada até a $12^{\text {a }}$ semana gestacional, com realização de no mínimo seis consultas (valor corrigido conforme idade gestacional no momento do parto), registro no cartão de pré-natal de pelo menos um resultado de cada exame de rotina e recebimento de orientação para maternidade de referência ${ }^{10}$.

A variável sexo do RN (masculino ou feminino) não foi incluída em nenhum nível de determinação hierárquica; porém, fez parte do modelo final por ser um importante preditor da mortalidade neonatal ${ }^{7}$.

Para a análise dos dados, inicialmente, foram estimadas as frequências absolutas e relativas das variáveis preditoras. A análise bivariada utilizou o teste qui-quadrado de Pearson, o risco relativo (RR) e intervalos de confiança (IC) a 95\% para avaliar a associação das variáveis. A análise multivariada utilizou modelos de regressão de Poisson com variância robusta para identificar as variáveis associadas ao near miss neonatal. O RR foi utilizado para analisar a associação das variáveis sociodemográficas, maternas e da organização dos serviços de saúde com o near miss neonatal. As variáveis com valor-p $<0,20$ na análise bivariada foram selecionadas para análise multivariada. Apenas as variáveis com valor-p $<0,05$ no modelo multivariado foram mantidas no modelo final. As variáveis colineares com fator de inflação da variância $<10$ foram excluídas do modelo.

O estudo principal foi aprovado pelo Comitê de Ética em Pesquisa (CEP) da Escola Nacional de Saúde Pública da Fundação Oswaldo Cruz (Parecer no 92/10; CAE: 0096.0.031.000-10). A presente pesquisa foi submetida ao CEP do Instituto Nacional de Saúde da Mulher, da Criança e do Adolescente Fernandes Figueira e aprovada pelo Parecer no 3.376.235 (CAAE: 14248719.1.0000.5269), cumprindo os preceitos da Resolução no 466/2012 do Conselho Nacional de Saúde ${ }^{18}$. Todas as mulheres participantes concederam suas entrevistas e informações por meio de um termo de consentimento livre e esclarecido.

\section{RESULTADOS}

Nesta pesquisa, 832 foi o número ponderado de $\mathrm{RN}$ que cumpriram os critérios de near miss neonatal, e 23.005 não o fizeram, totalizando $23.837 \mathrm{RN}$. Na Tabela 1, observa-se que o risco de ocorrência de near miss neonatal, quando comparadas as categorias sociodemográficas, foi maior entre as mulheres que cursaram o ensino fundamental incompleto $(4,2 \%)$, que se autodeclararam pretas/pardas/amarelas/indígenas $(4,1 \%)$ e que pertenciam à classe econômica C $(3,8 \%)$.

Quanto às características maternas (Tabela 2), o risco de near miss foi maior entre as mulheres que tiveram parto cesáreo (4,3\%), que declararam fazer uso de fumo (5,1\%), que tiveram síndrome hipertensiva da gestação $(8,7 \%)$, diabetes pré-gestacional $(12 \%)$ e gestacional $(4,4 \%)$. Além disso, foi observado maior risco de near miss neonatal nas mulheres com gravidez gemelar (11,8\%), quando comparadas às de gestação única (3,7\%). 
Tabela 1. Distribuição das condições sociodemográficas (nível distal) segundo near miss neonatal. Brasil, 2011-2012.

\begin{tabular}{|c|c|c|c|c|c|}
\hline Variáveis & $\%$ Total & $\%$ NMN & $\mathbf{R R}$ & IC95\% & $\mathbf{p}^{\mathbf{a}}$ \\
\hline \multicolumn{6}{|l|}{ Sexo } \\
\hline Masculino & 51,7 & 4,1 & 1,15 & $0,98-1,35$ & 0,078 \\
\hline Feminino & 48,3 & 3,5 & 1 & - & - \\
\hline \multicolumn{6}{|l|}{ Distal } \\
\hline \multicolumn{6}{|l|}{ Região } \\
\hline Sudeste & 42,6 & 4,3 & 1,38 & $0,99-1,92$ & 0,057 \\
\hline Norte & 9,5 & 3,1 & 0,99 & $0,63-1,56$ & 0,980 \\
\hline Nordeste & 28,8 & 3,5 & 1,12 & $0,74-1,69$ & 0,592 \\
\hline Sul & 12,5 & 3,1 & 1 & - & - \\
\hline Centro-Oeste & 6,6 & 4,1 & 1,31 & $0,86-2,01$ & 0,204 \\
\hline \multicolumn{6}{|l|}{ Escolaridade } \\
\hline Ensino fundamental incompleto & 26,5 & 4,2 & 1,18 & $0,82-1,69$ & 0,357 \\
\hline Ensino fundamental completo & 25,6 & 3,6 & 1,03 & $0,69-1,52$ & 0,875 \\
\hline Ensino médio completo & 38,9 & 3,7 & 1,04 & $0,68-1,59$ & 0,837 \\
\hline Ensino superior completo ou mais & 8,9 & 3,5 & 1 & - & - \\
\hline \multicolumn{6}{|l|}{ Raça/cor } \\
\hline Branca & 33,8 & 3,3 & 1 & - & - \\
\hline Preta/parda/amarela/indígena & 66,2 & 4,1 & 1,24 & $0,98-1,56$ & 0,075 \\
\hline \multicolumn{6}{|l|}{ Chefe de família } \\
\hline Não & 89,6 & 3,9 & 1 & - & - \\
\hline Sim & 10,4 & 3,0 & 0,76 & $0,57-1,02$ & 0,071 \\
\hline \multicolumn{6}{|l|}{ Classe econômicab } \\
\hline Classe $\mathrm{D}+\mathrm{E}$ & 23,6 & 3,8 & 1,14 & $0,89-1,47$ & 0,303 \\
\hline Classe C & 52,0 & 4,0 & 1,18 & $0,89-1,56$ & 0,249 \\
\hline Classe $\mathrm{A}+\mathrm{B}$ & 24,3 & 3,4 & 1 & - & - \\
\hline
\end{tabular}

NMN: near miss neonatal; RR: risco relativo; IC95\%: intervalo de confiança de 95\%.

a Teste qui-quadrado de Pearson.

${ }^{\mathrm{b}}$ Conforme classificação da Abep.

No bloco referente à organização dos serviços de saúde (Tabela 3), foi identificado maior risco de near miss neonatal nos filhos das mulheres que não realizaram o pré-natal adequado. Ademais, esse desfecho foi maior (5,5\%) quando o parto ocorreu na capital.

As Tabelas 1, 2 e 3 apresentam os resultados da análise bivariada para todas as variáveis independentes incluídas no modelo. No nível distal (Tabela 1), nenhuma variável foi associada ao desfecho. No nível intermediário (Tabela 2), foram associadas ao near miss neonatal as seguintes variáveis: idade maior ou igual a 35 anos ( $R R=1,51$; IC95\% 1,23-1,85), parto cesáreo ( $\mathrm{RR}=1,36$; IC95\% 1,10-1,67), síndrome hipertensiva gestacional $(\mathrm{RR}=2,71$; IC95\% 2,21-3,33), diabetes pré-gestacional ( $\mathrm{RR}=3,23$; IC:95\% 1,90-5,30) e gestação gemelar (RR = 3,18; IC95\% 2,25-4,50). Não houve associação entre o diabetes mellitus gestacional e o near miss neonatal. No nível proximal (Tabela 3), foram associadas as seguintes variáveis: pré-natal não adequado $(\mathrm{RR}=1,66$; IC95\% $1,32-2,08)$ e realização do parto na capital $(\mathrm{RR}=1,95$; IC95\% 1,44-2,65).

A Tabela 4 apresenta o modelo de regressão multivariada. Verificou-se associação significativa entre o near miss neonatal e as seguintes variáveis: idade da mãe maior ou igual a 35 anos ( $\mathrm{RR}=1,32$; IC95\% 1,04-1,66), parto cesáreo ( $\mathrm{RR}=1,34$; IC95\% 1,07-1,67), uso habitual de fumo ( $R R=1,48$; IC95\% 1,04-2,10), síndrome hipertensiva da gestação $(\mathrm{RR}=2,49 ; \mathrm{IC} 95 \%$ 1,98-3,14), diabetes pré-gestacional $(\mathrm{RR}=2,63$; IC95\% 1,36-5,05), gestação gemelar (RR = 2,98; IC95\% 1,90-4,68), pré-natal não adequado (RR = 1,71; IC95\% 1,36-2,16) e localização do hospital/maternidade na capital (RR = 1,89; IC95\% 1,40-2,55). 
Tabela 2. Distribuição das características maternas (nível intermediário) segundo near miss neonatal. Brasil, 2011-2012.

\begin{tabular}{|c|c|c|c|c|c|}
\hline Variáveis & $\%$ Total & $\%$ NMN & $\mathbf{R R}$ & IC95\% & p* \\
\hline \multicolumn{6}{|l|}{ Sexo } \\
\hline Masculino & 51,7 & 4,1 & 1,15 & $0,98-1,35$ & 0,078 \\
\hline Feminino & 48,3 & 3,5 & 1 & - & - \\
\hline \multicolumn{6}{|l|}{ Intermediário } \\
\hline \multicolumn{6}{|l|}{ Idade (anos) } \\
\hline 12 a 19 & 19,1 & 4,3 & 1,24 & $0,95-1,62$ & 0,107 \\
\hline 20 a 34 & 70,5 & 3,4 & 1 & - & - \\
\hline$\geq 35$ & 10,4 & 5,2 & 1,51 & $1,23-1,85$ & $<0,001$ \\
\hline \multicolumn{6}{|l|}{ Situação conjugal } \\
\hline Sem companheiro & 18,5 & 4,3 & 1,18 & $0,94-1,46$ & 0,138 \\
\hline Com companheiro & 81,5 & 3,7 & 1 & - & - \\
\hline \multicolumn{6}{|l|}{ Primípara } \\
\hline Não & 53,3 & 3,6 & 1 & - & - \\
\hline Sim & 46,7 & 4,0 & 1,12 & $0,94-1,35$ & 0,207 \\
\hline \multicolumn{6}{|l|}{ Tipo de parto } \\
\hline Vaginal & 46,6 & 3,2 & 1 & - & - \\
\hline Fórceps & 1,4 & 5,4 & 1,70 & $0,88-3,31$ & 0,115 \\
\hline Cesariana & 52,0 & 4,3 & 1,36 & $1,10-1,67$ & 0,004 \\
\hline \multicolumn{6}{|l|}{ Uso habitual de fumo } \\
\hline Não & 92,8 & 3,7 & 1 & - & - \\
\hline Sim & 7,2 & 5,1 & 1,38 & $0,97-1,96$ & 0,069 \\
\hline \multicolumn{6}{|c|}{ Síndromes hipertensivas gestacionais } \\
\hline Não & 89,0 & 3,2 & 1 & - & - \\
\hline Sim & 11,0 & 8,7 & 2,71 & $2,21-3,33$ & $<0,001$ \\
\hline \multicolumn{6}{|l|}{ Sífilis } \\
\hline Não & 99,0 & 3,8 & 1 & - & - \\
\hline Sim & 1,0 & 4,9 & 1,31 & $0,65-2,64$ & 0,448 \\
\hline \multicolumn{6}{|l|}{ Diabetes pré-gestacional } \\
\hline Não & 99,0 & 3,7 & 1 & - & - \\
\hline Sim & 1,0 & 12,0 & 3,23 & $1,9-5,3$ & $<0,001$ \\
\hline \multicolumn{6}{|l|}{ Diabetes gestacional } \\
\hline Não & 91,8 & 3,8 & 1 & - & - \\
\hline Sim & 8,2 & 4,4 & 1,18 & $0,92-1,52$ & 0,197 \\
\hline \multicolumn{6}{|c|}{ Suspeição de uso inadequado do álcool } \\
\hline Não há suspeita & 3,9 & 4,3 & 1,16 & $0,66-2,03$ & 0,609 \\
\hline Suspeita de uso & 10,0 & 4,5 & 1,23 & $0,94-1,61$ & 0,126 \\
\hline Não ingeriu álcool & 86,1 & 3,7 & 1 & - & - \\
\hline \multicolumn{6}{|l|}{ Tipo de gestação } \\
\hline Única & 98,8 & 3,7 & 1 & - & - \\
\hline Gemelar & 1,2 & 11,8 & 3,18 & $2,25-4,50$ & $<0,001$ \\
\hline
\end{tabular}

NMN: near miss neonatal; RR: risco relativo; IC95\%: intervalo de confiança de $95 \%$.

* Teste qui-quadrado de Pearson.

Tabela 3. Distribuição da organização do serviço de saúde (nível proximal) segundo near miss neonatal. Brasil, 2011-2012.

\begin{tabular}{|c|c|c|c|c|c|}
\hline Variáveis & $\%$ Total & $\%$ NMN & $\mathbf{R R}$ & IC95\% & $\mathrm{p}^{*}$ \\
\hline \multicolumn{6}{|l|}{ Sexo } \\
\hline Masculino & 51,7 & 4,1 & 1,15 & $0,98-1,35$ & 0,078 \\
\hline Feminino & 48,3 & 3,5 & 1 & - & - \\
\hline \multicolumn{6}{|l|}{ Proximal } \\
\hline \multicolumn{6}{|c|}{ Pré-natal adequado } \\
\hline Não & 36,7 & 5,1 & 1,66 & $1,32-2,08$ & $<0,001$ \\
\hline Sim & 63,3 & 3,1 & 1 & - & - \\
\hline \multicolumn{6}{|c|}{ Local das consultas pré-natais } \\
\hline Público & 70,7 & 4,0 & 1,27 & $1,00-1,62$ & 0,050 \\
\hline Particular & 29,3 & 3,1 & 1 & - & - \\
\hline \multicolumn{6}{|c|}{ Localização do hospital/maternidade } \\
\hline Não capital & 63,4 & 2,8 & 1 & - & - \\
\hline Capital & 36,6 & 5,5 & 1,95 & $1,44-2,65$ & $<0,001$ \\
\hline
\end{tabular}

NMN: near miss neonatal; RR: risco relativo; IC95\%: intervalo de confiança de 95\%.

* Teste qui-quadrado de Pearson. 
Tabela 4. Regressão multivariada das condições sociodemográficas, das características maternas e da organização dos serviços de saúde segundo near miss neonatal. Brasil, 2011-2012.

\begin{tabular}{|c|c|c|c|}
\hline Variáveis & RR ajustado & IC95\% & $\mathbf{p}^{*}$ \\
\hline \multicolumn{4}{|l|}{ Sexo do recém-nascido } \\
\hline Masculino & 1,18 & $1,00-1,40$ & 0,054 \\
\hline Feminino & 1 & - & - \\
\hline \multicolumn{4}{|l|}{ Distal } \\
\hline \multicolumn{4}{|l|}{ Raça/cor } \\
\hline Branca & 1 & - & - \\
\hline Preta/parda/amarela/indígena & 1,21 & $0,95-1,55$ & 0,126 \\
\hline \multicolumn{4}{|l|}{ Intermediário } \\
\hline \multicolumn{4}{|l|}{ Idade (anos) } \\
\hline 12 a 19 & 1,28 & $0,98-1,67$ & 0,073 \\
\hline 20 a 34 & 1 & - & - \\
\hline$\geq 35$ & 1,32 & $1,04-1,66$ & 0,020 \\
\hline \multicolumn{4}{|l|}{ Tipo de parto } \\
\hline Vaginal & 1 & - & - \\
\hline Fórceps & 1,75 & $0,81-3,77$ & 0,151 \\
\hline Cesariana & 1,34 & $1,07-1,67$ & 0,009 \\
\hline \multicolumn{4}{|l|}{ Uso habitual de fumo } \\
\hline Não & 1 & - & - \\
\hline Sim & 1,48 & $1,04-2,10$ & 0,031 \\
\hline \multicolumn{4}{|c|}{ Síndromes hipertensivas gestacionais } \\
\hline Não & 1 & - & - \\
\hline Sim & 2,49 & $1,98-3,14$ & $<0,001$ \\
\hline \multicolumn{4}{|l|}{ Diabetes pré-gestacional } \\
\hline Não & 1 & & \\
\hline Sim & 2,63 & $1,36-5,05$ & 0,004 \\
\hline \multicolumn{4}{|l|}{ Tipo de gestação } \\
\hline Única & 1 & - & - \\
\hline Gemelar & 2,98 & $1,90-4,68$ & $<0,001$ \\
\hline \multicolumn{4}{|l|}{ Proximal } \\
\hline \multicolumn{4}{|l|}{ Pré-natal adequado } \\
\hline Não & 1,71 & $1,36-2,16$ & $<0,001$ \\
\hline Sim & 1 & - & - \\
\hline \multicolumn{4}{|c|}{ Localização do hospital/maternidade } \\
\hline Não capital & 1 & - & - \\
\hline Capital & 1,89 & $1,40-2,55$ & $<0,001$ \\
\hline
\end{tabular}

RR: risco relativo; IC95\%: intervalo de confiança de 95\%.

* Teste qui-quadrado de Pearson.

\section{DISCUSSÃO}

Os resultados revelaram a proeminência das características maternas (nível intermediário) na determinação do near miss neonatal, com importante contribuição das condições assistenciais recebidas no pré-natal (nível proximal), consideradas fatores passíveis de intervenção ${ }^{10}$.

A faixa etária materna igual ou superior a 35 anos, considerada fator de risco para inúmeros desfechos negativos relacionados aos $\mathrm{RN}^{19,20}$, teve sua associação ao near miss neonatal confirmada nesta pesquisa, corroborando outros estudos da literatura ${ }^{2,8}$. O estudo de coorte prospectiva de nascimento em seis maternidades brasileiras conduzido por Kale et al. ${ }^{8}$ observou que os RN de mães com idade materna avançada apresentaram quase duas vezes mais risco de near miss neonatal. As mulheres com idade superior a 35 anos têm maior frequência de resultados perinatais adversos quando comparadas às mulheres com idade 
entre 20 e 34 anos, com destaque para prematuridade, baixo peso ao nascer e baixo índice de Apgar ${ }^{20}$. Além disso, os filhos dessas mulheres correm maior risco de morrer no período neonatal devido às complicações obstétricas secundárias a doenças pré-existentes ${ }^{21}$.

O parto cesáreo se manteve associado à ocorrência de near miss neonatal neste estudo, resultado já apontado na literatura da área ${ }^{2,5,8}$. Silva et al. ${ }^{2}$, também na pesquisa Nascer no Brasil, observaram que a chance de near miss neonatal foi duas vezes maior entre as mulheres que realizaram cesáreas, ou seja, essa variável aparece como fator de risco para tal desfecho, mas também como fator de proteção para a mortalidade neonatal, dado que as crianças nascidas de parto vaginal apresentaram maior taxa de mortalidade neonatal. Assim, a via de parto em si não seria causa da ocorrência de complicações materno-fetais, mas sim a indicação clínica de cesariana. Para elucidar esse ponto, seria necessário investigar se a indicação de cesárea era intraparto, devido a complicações materno-fetais, ou eletiva, sem qualquer base clínica5.

A aplicação do modelo hierarquizado nesta investigação apontou que, dentre os fatores maternos analisados, o uso habitual de fumo após o quinto mês de gestação foi associado ao aumento do risco de near miss neonatal. Os efeitos adversos do fumo materno durante a gravidez incidem sobre o peso do RN. Todavia, o fumo é um dos determinantes modificáveis mais importantes para minimizar o risco de baixo peso ao nascer e de outros resultados perinatais adversos ${ }^{15}$. O impacto negativo do tabagismo materno durante toda a gestação no comprimento e no perímetro cefálico do RN indica que tal comportamento tem uma relação linear inversa com essas dimensões: quanto maior o tempo de gestação com exposição ao fumo, menores serão as medidas antropométricas do $\mathrm{RN}^{22}$. Esses achados foram apontados em uma coorte de base populacional com 8.621 nascidos vivos europeus, na qual se observou que, desde o início do segundo trimestre até o término da gravidez, os fetos de mulheres que continuaram a fumar pesaram menos em relação aos das não fumantes. Mais especificamente, a diferença de peso prevista nos filhos de mulheres fumantes na $20^{\mathrm{a}}$ semana (IC95\%) foi de $-2,6 \mathrm{~g}(-5,1 \mathrm{a}-0,1)$, e na $40^{\mathrm{a}}$ semana gestacional foi de $-207 \mathrm{~g}(-231 \mathrm{a}-182)^{15}$.

Em relação às doenças crônicas, foi observado que as mulheres com síndrome hipertensiva da gestação apresentaram o dobro de risco de near miss neonatal. De forma semelhante, Oliveira et al. ${ }^{23}$ e Nardello et al. ${ }^{19}$, em estudos transversais em maternidades de Recife e Sergipe, respectivamente, observaram que a hipertensão gestacional foi fortemente associada a desfechos neonatais adversos. Apesar de inúmeros fatores e teorias sugeridas para explicar as possíveis causas desse agravo, a etiologia da síndrome hipertensiva na gestação ainda é pouco conhecida ${ }^{17,24}$; no entanto, seus efeitos têm sido associados a prematuridade, Apgar baixo e asfixia neonatal ${ }^{25}$.

Na mesma linha de raciocínio, também foi observada associação entre diabetes mellitus pré-gestacional e near miss neonatal. O aumento da prevalência de diabetes mellitus pré-gestacional e gestacional nos últimos anos pode ser justificado pela epidemia de obesidade, pelo aumento da idade materna e pela detecção precoce da doença, considerando a maior cobertura do pré-natal e a diminuição do ponto de corte do diagnóstico da diabetes gestacional (glicemia em jejum reduzida de $92 \mathrm{mg} / \mathrm{dL}$ para $85 \mathrm{mg} / \mathrm{dL})^{26}$. Assim como a síndrome hipertensiva da gestação, a presença de diabetes mellitus na gestação também está associada a elevado risco de morbimortalidade neonatal ${ }^{27}$. Alguns estudos ${ }^{25,27}$ se detiveram sobre a avaliação da associação entre diabetes pré-gestacional e alguns desfechos neonatais, sobretudo prematuridade, anomalias congênitas - como más-formações cardiovasculares -, asfixia perinatal, angústia respiratória e complicações metabólicas (hipoglicemia, hipocalcemia, policitemia e hiperbilirrubinemia). Ainda que esses estudos não tenham avaliado o efeito da doença sobre o near miss neonatal, as citadas repercussões negativas do diabetes mellitus pré-gestacional sobre a saúde do RN mostram uma possível elucidação de seus efeitos sobre esse desfecho.

Houve um risco aproximadamente três vezes maior de near miss neonatal para gestação gemelar neste estudo. Tal resultado revela que o parto gemelar - condição rara que apresenta 
diversas particularidades e dificuldades, não apenas no manejo clínico, mas também na abordagem científica - ainda é considerado um desafio para o serviço de saúde e para as investigações sobre maiores riscos maternos e perinatais ${ }^{28}$. Vale destacar que a gestação gemelar aumenta a taxa de mortalidade perinatal de duas a três vezes, principalmente devido ao nascimento prematuro, à restrição de crescimento intrauterino, ao baixo peso ao nascer e à anóxia intraparto ${ }^{29,30}$. Portanto, é de extrema importância que haja atendimento pré-natal adequado visando resultados maternos e perinatais melhores nessa condição.

A falta de acesso e a qualidade do pré-natal são determinantes notáveis para a ocorrência de near miss neonatal ${ }^{5,8}$. Neste estudo, a não adequação do pré-natal (nível proximal) foi associada a esse desfecho, aumentando seu risco. Embora a assistência pré-natal no Brasil tenha alcançado cobertura praticamente universal, persistem desigualdades no acesso ao cuidado adequado ${ }^{10,11}$. Ressalta-se que o acompanhamento pré-natal possibilita a detecção e o tratamento precoce de afecções maternas pré-existentes e/ou iniciadas no período gestacional, bem como de alterações no concepto, reduzindo o risco de complicações obstétricas e morte neonatal devido a prematuridade, más-formações ou infecções congênitas, as quais constituem as causas mais frequentes de morte neonatal no mundo ${ }^{9,10}$. Vale informar que o número adequado de consultas pré-natais (seis ou mais) não garante por si só a qualidade da assistência materno-infantil, sendo necessário assegurar o início precoce do pré-natal (até a $12^{\mathrm{a}}$ semana de gestação), a assistência por profissionais qualificados, a existência de recursos físicos e materiais adequados, a realização dos exames preconizados e o tratamento oportuno, caso necessário ${ }^{17,21,25,26}$.

Em relação à localização do hospital/maternidade para a realização do parto, observou-se que o risco de ocorrência de near miss neonatal quase dobrou entre os RN que nasceram nas capitais. Esse resultado pode ser parcialmente explicado pelo fato de as não capitais terem menor oferta de serviços especializados para atendimento de alto risco ${ }^{2}$, com condições menos adequadas ao atendimento de gestantes nesse quadro. Assim, as capitais ficam sendo referência para gestantes residentes em não capitais que apresentam complicações na gravidez ${ }^{10}$. Ressalta-se que a gravidade da doença parece ser um fator de confusão na associação entre hospital de nascimento e near miss neonatal. A alta disponibilidade de UTI neonatais e a precoce intervenção médica em grandes centros urbanos são fatores apontados por Silva et al. ${ }^{2}$ como possíveis justificativas para a maior ocorrência do near miss neonatal nas capitais. $O$ presente estudo não teve como objetivo analisar a gravidade dos casos de near miss neonatal, impossibilitando, assim, apontar se a situação dos RN se agravava antes ou após o tratamento.

A principal limitação do estudo foi o fato de não ter considerado os hospitais com menos de 500 partos e os nascidos em domicílio. Destaca-se que o início de uma segunda versão da pesquisa Nascer no Brasil está previsto para 2020, mas até o presente momento não foram encontrados artigos com dados de near miss neonatal em nível nacional.

Todavia, este estudo apresenta como vantagem ter sido realizado a partir de uma base de dados hospitalar representativa da população brasileira. Além disso, oferece uma análise hierarquizada da determinação do near miss neonatal, com um amplo conjunto de variáveis para a avaliação da saúde das gestantes brasileiras, permitindo analisar as inter-relações envolvidas na rede de causalidade desse desfecho. Portanto, a identificação das variáveis que exercem maior impacto na ocorrência do near miss neonatal possibilita a adoção de medidas de prevenção e de intervenção na assistência pré-natal das gestantes, repercutindo na saúde de seus RN.

\section{CONCLUSÕES}

Embora características passíveis de intervenção pelo aconselhamento - como o uso habitual de fumo - tenham se associado à ocorrência do near miss neonatal, outros fatores determinantes desse desfecho se referiam à prestação dos serviços e dos cuidados. Portanto, 
é de extrema importância enfatizar a adequação do pré-natal para a identificação das gestantes que necessitam de atenção mais especializada, com acompanhamento oportuno durante a gravidez, o parto e o pós-parto para a prevenção de condições perinatais que ameaçam a vida.

\section{REFERÊNCIAS}

1. Santos JP, Pileggi-Castro C, Camelo Jr JS, Silva AA, Duran P, Serruya JS et al. Neonatal near miss: a systematic review. BMC Pregnancy Childbirth. 2015;15:320. https://doi.org/10.1186/s12884-015-0758-y

2. Silva AAM, Leite AJM, Lamy ZC, Moreira MEL, Gurgel RQ, Cunha AJLA, et al. Neonatal near miss in the Birth in Brazil survey. Cad Saude Publica. 2014;30 Supl 1:S1-10. https://doi.org/10.1590/0102-311X00129613

3. Kale PL, Mello-Jorge MHP, Laurenti R, Fonseca SC, Silva KS. Pragmatic criteria of the definition of neonatal near miss: a comparative study. Rev Saude Publica. 2017;51:111. https://doi.org/10.11606/S1518-8787.2017051006587

4. França KEX, Vilela MBR, Frias PG, Gaspar GS, Sarinho SW. Early neonatal near miss identified through health information systems. Cad Saude Publica. 2018;34(9): e00167717. https://doi.org/10.1590/0102-311x00167717

5. Silva GA, Rosa KA, Saguier ESF, Henning E, Mucha F, Franco SC. Estudo de base populacional sobre a prevalência de near miss neonatal em município do sul do Brasil: prevalência e fatores associados. Rev Bras Saude Matern Infant. 2017;17(1):159-67. https://doi.org/10.1590/1806-93042017000100009

6. Leal MC, Szwarcwald CL, Almeida PVB, Aquino EML, Barreto ML, Barros F, et al. Saúde reprodutiva, materna, neonatal e infantil nos 30 anos do Sistema Único de Saúde (SUS). Cienc Saude Coletiva. 2018;23(6):1915-28. https://doi.org/10.1590/1413-81232018236.03942018

7. Wang $\mathrm{H}$, Liddell CA, Coates MM, Mooney MD, Levitz CE, Schumacher AE, et al. Global, regional, and national levels of neonatal, infant, and under-5 mortality during 1990-2013: a systematic analysis for the Global Burden of Disease Study 2013. Lancet. 2014;384(9947):957-79. https://doi.org/10.1016/S0140-6736(14)60497-9

8. Kale PL, Mello-Jorge MHP, Silva KS, Fonseca SC. Neonatal near miss and mortality: factors associated with life-threatening conditions in newborns at six public maternity hospitals in Southeast Brazil. Cad Saude Publica. 2017;33(4):e00179115. https://doi.org/10.1590/0102-311x00179115

9. Garcia LP, Santana LR. Evolução das desigualdades socioeconômicas na mortalidade infantil no Brasil, 1993-2008. Cienc Saude Coletiva. 2011;16(9):3717-28. https://doi.org/10.1590/S1413-81232011001000009

10. Veloso FCS, Kassar LML, Oliveira MJC, Lima THB, Bueno NB, Gurgel RQ, et al. Análise dos fatores de risco na mortalidade neonatal no Brasil: uma revisão sistemática e metanálise de estudos observacionais. J Pediatr (Rio J.). 2019;95(5):519-30. https://doi.org/10.1016/j.jped.2018.12.014

11. Vasconcellos MTL, Silva PLN, Pereira APE, Schilithz AOC, Souza Junior PRB, Szwarcwald CL. Sampling design for the Birth in Brazil: National Survey into Labor and Birth. Cad Saude Publica. 2014;30 Supl 1:S1-10. https://doi.org/10.1590/0102-311X00176013

12. Leal MC, Silva AAM, Dias MAB, Gama SGN, Rattner D, Moreira ME, et al. Birth in Brazil: national survey into labour and birth. Reprod Health. 2012;9:15. https://doi.org/10.1186/1742-4755-9-15

13. Lima S, Carvalho ML, Vasconcelos AGG. Proposta de modelo hierarquizado aplicado à investigação de fatores de risco de óbito infantil neonatal. Cad Saude Publica. 2008;24(8):1910-6. https://doi.org/10.1590/S0102-311X2008000800019

14. Associação Brasileira de Empresas de Pesquisa. Critério de Classificação Econômica Brasil; 2008. São Paulo: ABEP; 2008 [citado 4 nov 2018]. Disponível em: https://www.abep.org

15. Brand JS, Gaillard R, West J, McEachan RRC, Wright J, Voerman E, et al. Associations of maternal quitting, reducing and continuing smoking during pregnancy with longitudinal fetal growth: findings from Mendelian randomization and parental negative control studies. PLoS Med. 2019;16(11):e1002972. https://doi.org/10.1371/journal.pmed.1002972 
16. Sokol RJ, Martier SS, Ager JW. The T-ACE questions: practical prenatal detection of risk-drinking. Am J Obstet Gynecol. 1989;160(4):863-8. https://doi.org/10.1016/0002-9378(89)90302-5

17. Nascimento TLC, Bocardi MIB, SantaRosa MPR. Doença Hipertensiva Específica da Gravidez (DHEG) em adolescentes: uma revisão de literatura. Ideias Inov. 2015;2(2):69-76

18. Ministério da Saúde (BR), Conselho Nacional de Saúde. Resolução nº 466, de 12 de dezembro de 2012. Aprova as diretrizes e normas regulamentadoras de pesquisas envolvendo seres humanos. Diário Oficial da União. 13 jun. 2013; Seção 1:59.

19. Nardello DM, Guimarães AMDN, Barreto IDC, Gurgel RQ, Ribeiro ERO, Gois CFL. Óbitos fetais e neonatais de filhos de pacientes classificadas com near miss. Rev Bras Enferm. 2017;70(1):104-11. https://doi.org/10.1590/0034-7167-2016-0405

20. Laopaiboon M, Lumbiganon P, Intarut N, Mori R, Ganchimeg T, Vogel JP, et al. Advanced maternal age and pregnancy outcomes: a multicountry assessment. BJOG. 2014;121 Suppl 1:49-56. https://doi.org/10.1111/1471-0528.12659

21. Amaral E, Souza JP, Surita F, Luz AG, Sousa MH, Cecatti JG, et al. A population-based surveillance study on severe acute maternal morbidity (near miss) and adverse perinatal outcomes in Campinas, Brazil: the Vigimoma Project. BMC Pregnancy Childbirth. 2011;11:9. https://doi.org/10.1186/1471-2393-11-9

22. Zhang L, González-Chica DA, Cesar JA, Mendoza-Sassi RA, Beskow B, Larentis N, et al. Tabagismo materno durante a gestação e medidas antropométricas do RN: um estudo de base populacional no extremo sul do Brasil. Cad Saude Publica. 2011;27(9):1768-76. https://doi.org/10.1590/S0102-311X2011000900010

23. Oliveira LC, Costa AAR. Óbitos fetais e neonatais entre casos de near miss materno. Rev Assoc Med Bras. 2013;59(5):487-94. https://doi.org/10.1016/j.ramb.2013.08.004

24. Dadelszen P, Payne B, Li J, Ansermino JM, Pipkin FB, Coté AM, et al. Prediction of adverse maternal outcomes in pre-eclampsia: development and validation of the fullPIERS model. Lancet. 2011;377(9761):219-27. https://doi.org/10.1016/s0140-6736(10)61351-7

25. Law A, McCoy M, Lynen R, Curkendall SM, Gatwood J, Juneau PL, et al. The prevalence of complications and healthcare costs during pregnancy. J Med Econ. 2015;18(7):533-41. https://doi.org/10.3111/13696998.2015.1016229

26. Negrato CA, Montenegro Jr RM, Mattar R, Zajdenverg L, Francisco RPV, Pereira BG, et al. Dysglycemias in pregnancy: from diagnosis to treatment. Brazilian consensus statement. Diabetol Metab Syndr. 2010;2:27. https://doi.org/10.1186/1758-5996-2-27

27. American Diabetes Association. Diagnosis and classification of diabetes mellitus. Diabetes Care. 2010;33 Suppl 1:S62-9. https://doi.org/10.2337/dc10-S062

28. Santana DS, Surita FG, Cecatti JG. Multiple pregnancy: epidemiology and association with maternal and perinatal morbidity. Rev Bras Ginecol Obstet. 2018;40(9):554-62. https://doi.org/10.1055/s-0038-1668117

29. Obiechina NJ, Okolie V, Eleje GU, Okechukwu ZC, Anemeje OA. Twin versus singleton pregnancies: the incidence, pregnancy complications, and obstetric outcomes in a Nigerian tertiary hospital. Int J Womens Health. 2011;3:227-30. https://doi.org/10.2147/IJWH.S22059

30. Vogel JP, Torloni MR, Seuc A, Betrán AP, Widmer M, Souza JP, et al. Maternal and perinatal outcomes of twin pregnancy in 23 low- and middle-income countries. PLoS One. 2013;8(8):e70549. https://doi.org/10.1371/journal.pone.0070549

Contribuição dos Autores: Concepção e planejamento do estudo: TGP, DRM, VMF. Coleta, análise e interpretação dos dados: TGP, DRM, VMF, SNGG. Elaboração ou revisão do manuscrito: TGP, DRM, VMF, SNGG, MELM. Aprovação da versão final: TGP, DRM, VMF, SNGG, MELM. Responsabilidade pública pelo artigo: TGP, DRM, VMF.

Conflito de Interesses: Os autores declaram não haver conflito de interesses. 